PoS $\quad \begin{aligned} & \text { PROCEEDINGS } \\ & \text { OF SCIENCE }\end{aligned}$

\title{
Measurement of $\mathrm{Y}(\mathrm{nS})$ production at $7 \mathrm{TeV}$ with the CMS experiment
}

\author{
Herbert Rohringer* \\ for the CMS Collaboration, Institute for High Energy Physics,Austrian Academy of \\ Sciences, Nikolsdorfergasse 18,A-1050 Vienna,Austria \\ E-mail: herbert.rohringerdoeaw.ac.at
}

The production cross sections of $\mathrm{Y}(1 S), \mathrm{Y}(2 S)$, and $\mathrm{Y}(3 S)$ in proton-proton collisions at sqrt $(s)$ $=7 \mathrm{TeV}$ are measured using a data sample collected with the CMS detector at the LHC.

The 2011 Europhysics Conference on High Energy Physics-HEP 2011,

July 21-27, 2011

Grenoble, Rhône-Alpes, France

${ }^{*}$ Speaker. 
Hadroproduction and polarization of heavy quarkonia is in non relativistic QCD theoretically not clearly understood and modelled. Especially the simultaneous description of cross sections and polarization cannot be established experimentally. It is an open question, wether gluon gluon fusion of color singlet or color octet bottom pairs are main contributions for the production . Y $(1 S)$ is also a good candle for calibration,alignment and resolution measurements of a detector. The LHC experiment CMS is well suited to investigate the 3 known upsilon states via their 2 muon decays with rather good mass resolution due to an excellent muon system . Total and partial cross sections vs $p_{t}$ and rapidity y have been measured with 2010 data of an integrated luminosity of approximately $3.1 \mathrm{pb}^{-1}$.

The measurement procedure consists of determining the signal yields of the 3 upsilon resonances $\mathrm{Y}(1 S), \mathrm{Y}(2 S), \mathrm{Y}(3 S)$ via a simultaneous fit with Crystal ball functions to take into account the final state radiation and 2 nd order polynomials for the background in several ( $p_{t}$, rapidity ) bins. The calculation of the differential cross sections requires a careful determination of the acceptance and efficiency of the decay muons forming the upsilon states. The acceptance comes from MC studies by comparing generated and reconstructed muons in ( $p_{t}$, rapidity ) bins. Due to the limited acceptance of the detector only muons with minimum $p_{t}$ of $3.5 \mathrm{GeV} / \mathrm{c}$ for $|\eta|<1.6$ and $p_{t}$ of 2.5 for $|\eta|$ between 1.6 and 2.4 can be used. The total efficiency is supposed to be factorizable into the trigger efficiency, the efficiency to identify a track in the muon chambers as a $\mu$ and the efficiency, that an accepted muon track is succesfully reconstructed. All of them are determined with the tag and probe method using $\mathrm{J} / \psi(3.1)$ as the reference for fitting.

The 3 upsilon acceptances are varying in ( $p_{t}$, rapidity), because the 2 decay muons cannot always be reconstructed. Therefore the upsilons have a very small acceptance around a $p_{t}$ of $5 \mathrm{GeV} / \mathrm{c}$ and are also dependent on the $\mathrm{Y}(n S)$ masses.

The total cross sections are determined assuming 5 polarization schemes to find the uncertainty.

The results for the 3 upsilon states - for rapidities $|y|<2$. and the 'no polarization scheme' - are :

$$
\begin{aligned}
& \sigma(\mathrm{pp}->\mathrm{Y}(1 \mathrm{~S}) \mathrm{X} \cdot \mathrm{B}(\mathrm{Y}(1 \mathrm{~S}))->\mu \mu)=7.37 \pm 0.13 \text { (stat) } \\
& \sigma(\mathrm{pp}->\mathrm{Y}(2 \mathrm{~S}) \mathrm{X} \cdot \mathrm{B}(\mathrm{Y}(2 \mathrm{~S}))->\mu \mu)=1.90 \pm 0.09 \text { (stat) } \\
& \begin{array}{l}
+0.20 \\
-0.14
\end{array} \text { (syst) } \pm 0.24 \text { (lumi.) nb } \\
& \sigma(\mathrm{pp}->\mathrm{Y}(3 \mathrm{~S}) \mathrm{X} \cdot \mathrm{B}(\mathrm{Y}(3 \mathrm{~S}))->\mu \mu)=1.02 \pm 0.07 \text { (stat) }{ }_{-0.08}^{+0.11} \text { (syst) } \pm 0.11 \text { (lumi.) nb }
\end{aligned}
$$

We also measured cross section ratios :

$$
\begin{aligned}
& \mathrm{Y}(2 \mathrm{~S}) / \mathrm{Y}(1 \mathrm{~S})=0.26 \pm 0.02 \text { (stat) } \pm 0.04 \text { (syst) } \\
& \mathrm{Y}(3 \mathrm{~S}) / \mathrm{Y}(1 \mathrm{~S})=0.14 \pm 0.01 \text { (stat) } \pm 0.02 \text { (syst) }
\end{aligned}
$$

These cross section ratios $\mathrm{Y}(2 \mathrm{~S}) / \mathrm{Y}(1 \mathrm{~S})$ and $\mathrm{Y}(3 \mathrm{~S}) / \mathrm{Y}(1 \mathrm{~S})$ are also found to be rising in $p_{t}$.

\section{References}

[1] CMS Collaboration Measurement of the inclusive upsilon production cross section in pp collisions at $\operatorname{sqrt}(s)=7$ TeV, Phys Rev D 83, 2011(112004) 